\title{
Cosmology calculations almost without general relativity
}

\author{
Thomas F. Jordan \\ Physics Department \\ University of Minnesota \\ Duluth, Minnesota 55812 \\ tjordan@d.umn.edu
}

\begin{abstract}
The Friedmann equation is derived for a Newtonian universe. Changing mass density to energy density gives exactly the Friedmann equation of general relativity. Accounting for work done by pressure then yields the two Einstein equations that govern the expansion of the universe. Descriptions and explanations of radiation pressure and vacuum pressure are added to complete a basic kit of cosmology tools. It provides a basis for teaching cosmology to undergraduates in a way that quickly equips them to do basic calculations. This is demonstrated with calculations involving: characteristics of the expansion for densities dominated by radiation, matter, or vacuum; the closeness of the density to the critical density; how much vacuum energy compared to matter energy is needed to make the expansion accelerate; and how little is needed to make it stop. Travel time and luninosity distance are calculated in terms of the redshift and the densities of matter and vacuum energy, using a scaled Friedmann equation with the constant in the curvature term determined by matching with the present values of the Hubble parameter and energy density. General relativity is needed only for the luminosity distance, to describe how the curvature of space, determined by the energy density, can change the intensity of light by changing the area of the sphere to which the light has spread. Thirty-one problems are included.
\end{abstract}




\section{INTRODUCTION}

To understand cosmology, it is necessary to work with equations that come from general relativity. That does not mean a student needs a course in general relativity to get started. Substantial working knowledge of cosmology can be built on simple undergraduate physics. The Friedmann equation is the key. It is one of the two Einstein equations of general relativity that govern the expansion of the universe. The other can be easily understood; it accounts for work done by pressure as the universe expands.

The Friedmann equation can be derived for a Newtonian universe where motion is nonrelativistic and gravity is attraction between masses. ${ }^{1}$ In this case, the Friedmann equation describes conservation of nonrelativistic kinetic energy plus gravitational potential energy for the motion of a galaxy in the expansion. To move to relativity we use the equivalence of mass and energy and change mass density to energy density. What we need to know about general relativity at this step is that it gives exactly the same Friedmann equation in all cases. The foundation is curvature of space rather than gravitational energy, but the result is the same. There are no correction terms. The only change we have to make is to interpret the density in the Friedmann equation as that of energy rather than mass and include all forms of energy in it.

That is reviewed and developed here. Descriptions and explanations of radiation pressure and vacuum pressure are added to complete a basic kit of cosmology tools. It fills in the mathematics left out of descriptive reviews ${ }^{2}$ and provides a basis for teaching cosmology to undergraduates in a way that quickly equips them to do basic calculations. Application of the equations is demonstrated here with calculations that: determine characteristics of the expansion for cases where the energy density is dominated by radiation, matter, or vacuum; present and answer the question how the energy density got so close to the critical density; find how much vacuum energy compared to matter energy is needed to make the expansion accelerate; and find how little is needed to make it stop.

The travel time and luminosity distance for light from a distant source are calculated in terms of the redshift and the densities of matter and vacuum energy. In the scaled Friedmann equation that is used for this, the constant in the curvature term is determined by matching with the present values of the Hubble parameter and energy density and is found to be a function of them. General relativity is not needed for that, but it is needed to describe how the curvature of space can change the area of the sphere of light and, thereby, the intensity of the light spread over that sphere, and hence the luminosity distance used in Hubble plots. In particular, general relativity gives the curvature in terms of the energy density. Thirty-one problems, most of them easy, explore some of these topics in more detail and extend the range of application.

\section{FRIEDMANN EQUATION}

Two basic equations of cosmology govern the expansion of the universe. They can be understood as statements about energy. One says there is a constant like the sum of kinetic and gravitational energy for the motion of a galaxy in the expansion. The other equation accounts for the work done by pressure as the universe expands.

The expansion of the universe is observed as motion of galaxies away from each other. The universe is like an expanding gas, but the units are galaxies; an individual galaxy does not expand. The Hubble law describes what is observed. ${ }^{\mathbf{3}}$ The speeds of galaxies moving 
away from us are proportional to their distances from us. Galaxies at distances $R$ are moving away from us with average speed

$$
\frac{d R}{d t}=H R
$$

where $H$ is the Hubble parameter. The Hubble law is what we expect to observe in a spatially isotropic uniformly expanding universe. ${ }^{\mathbf{3}}$ We assume it would be the same for observers at any other location. We assume the universe is in fact homogeneous, the same everywhere, at any given time. Right now, that can be true only at scales larger than clusters of galaxies, but the isotropy of the cosmic radiation is evidence that at earlier times the universe was in fact very homogeneous. ${ }^{4}$ Gravity pulls the galaxies together and slows the expansion of the universe. If distances are measured from a typical galaxy, which could be at any location, the force of gravity on a galaxy at distance $R$ coming from the mass of homogeneous universe inside the sphere of radius $R$ is the same as if all that mass were at the center of the sphere. There is no force of gravity from the spherical shells of homogeneous universe outside that sphere. The kinetic plus gravitational potential energy for the motion of a galaxy of mass $m$ in the expansion is therefore

$$
E=\frac{1}{2} m\left(\frac{d R}{d t}\right)^{2}-\frac{G M m}{R}
$$

where $G$ is Newton's gravitational constant,

$$
M=\frac{4}{3} \pi R^{3} \rho
$$

is the mass inside the sphere of radius $R$, and $\rho$ is the density of mass in the universe. Using the Hubble law, Eq. (2.1), we get

$$
\frac{2 E}{m R^{2}}=H^{2}-\frac{8}{3} \pi G \rho
$$

At a given time in our homogeneous universe, $H$ and $\rho$ are constant throughout space. That means $2 E / m R^{2}$ is the same for all galaxies. In particular, the negative, zero, or positive character of $E$ is the same for all galaxies.

If $E$ is not zero, we can choose units and find a time $t_{1}$ so that $\left|2 E / m R\left(t_{1}\right)^{2}\right|$ is 1 . Then

$$
\frac{(d R / d t)^{2}}{R^{2}}-\frac{8}{3} \pi G \rho=-\frac{k R\left(t_{1}\right)^{2}}{R^{2}}
$$

where $k=-2 E / m R\left(t_{1}\right)^{2}$ is 1,0 or -1 depending on whether $E$ is negative, zero, or positive. The constant $R\left(t_{1}\right)^{2}$ is the magnitude of $2 E / m$. It stays constant while $R, d R / d t, H$ and $\rho$ change in time. (This constant will be handled in a completely different way in Sections IX and X where applications require its use. Meanwhile we will see how much can be done without knowing anything more about it.)

Now we invoke $E=m c^{2}$ and let $\rho$ be the energy density of the universe. We use units for which $c$ is 1 . Mass and energy are equivalent. We assume, as in general relativity, that all energy creates gravity. With $\rho$ the energy density, our Eq. (2.5) is the Friedmann equation obtained from general relativity, where $k$ is the constant 1,0 , or -1 that indicates whether the curvature of space is positive, zero, or negative. ${ }^{\mathbf{5}}$

In our Newtonian equations, $E$ is constant, so $k$ is constant. We have derived the Friedmann equation for the case of a Newtonian universe. General relativity says the Friedmann 
equation holds with constant $k R\left(t_{1}\right)^{2}$ in all cases. Of course general relativity provides a derivation of the Friedmann equation that is altogether more satisfactory. But Newtonian physics comes remarkably close. It gives us an equation that is exactly the Friedmann equation where Newtonian physics applies and with reinterpretation becomes exactly the Friedmann equation in all cases. It does not require correction terms.

\section{EINSTEIN EQUATIONS}

The Friedmann equation is one of the two Einstein equations of general relativity for the homogeneous universe. The other just adds that as a volume $V$ of the universe expands by an amount $d V$, the pressure $p$ in the volume does work $p d V$, which decreases the energy in the volume by that amount. So for a sphere of radius $R$,

$$
d\left(\rho \frac{4}{3} \pi R^{3}\right)=-p d\left(\frac{4}{3} \pi R^{3}\right)
$$

which implies that

$$
R \frac{d \rho}{d t}+3(\rho+p) \frac{d R}{d t}=0
$$

We can write the Friedmann Eq. (2.5) as

$$
\left(\frac{d R}{d t}\right)^{2}=\frac{8}{3} \pi G \rho R^{2}-k R\left(t_{1}\right)^{2} .
$$

Taking the time derivative of this, using Eq. (3.2) for $R d \rho / d t$, yields

$$
\frac{d^{2} R}{d t^{2}}=-\frac{4}{3} \pi G(\rho+3 p) R
$$

These last two Eqs. (3.3) and (3.4) are the two basic equations of cosmology obtained from general relativity. ${ }^{\mathbf{5}}$

\section{DENSITY AND PRESSURE}

The pressure depends on the nature of energy. The energy density can come from matter, radiation, vacuum, or combinations of these. Each gives a different pressure.

The energy density of matter does not produce pressure that affects the expansion of the universe. Galaxies are not colliding and creating pressure the way molecules in a gas do. In the early universe, the energy density was mostly from radiation. The density and pressure of radiation are related by

$$
p_{\gamma}=\frac{1}{3} \rho_{\gamma}
$$

That is shown in Appendix A.

The work done by each constituent decreases the energy of that constituent as the universe expands, so Eq. (3.1) holds separately for each constituent:

$$
\frac{d}{d t} \rho_{i} R^{3}=-p_{i} \frac{d}{d t} R^{3}
$$


where $i$ can be $m, \gamma$ or $v$ for matter, radiation, or vacuum. For matter, $p_{m}$ is zero, so

$$
\frac{d}{d t} \rho_{m} R^{3}=0 .
$$

The energy in a sphere of matter does not change as the sphere expands; it just spreads out. The time dependence of $\rho_{m}$ is tied to that of $R$ by the relation

$$
\rho_{m} \propto \frac{1}{R^{3}} .
$$

For radiation, Eqs. (4.1) and (4.2) give

$$
\begin{gathered}
\frac{d}{d t} \rho_{\gamma} R^{4}=\rho_{\gamma} R^{3} \frac{d R}{d t}+R \frac{d}{d t} \rho_{\gamma} R^{3} \\
=\rho_{\gamma} R^{3} \frac{d R}{d t}-R \frac{1}{3} \rho_{\gamma} \frac{d}{d t} R^{3}=0
\end{gathered}
$$

so the time dependence of $\rho_{\gamma}$ is determined by

$$
\rho_{\gamma} \propto \frac{1}{R^{4}} .
$$

As the universe expands, the wavelengths $\lambda$ of radiation expand in proportion to distances $R$. Since the wavelengths $\lambda$ and frequencies $\nu$ of radiation are related by

$$
\nu \lambda=c
$$

and the energy of a photon is

$$
E=h \nu,
$$

and since the average energy of a photon of radiation in equilibrium at temperature $T$ is $^{6}$

$$
\bar{E} \approx 2.7 k T
$$

where $h$ and $k$ are the Planck and Boltzmann constants, which we assume do not change, we see that the way wavelengths, frequencies, photon energies and temperature change as the universe expands is determined by

$$
\begin{gathered}
\lambda \propto R \\
E \propto \nu \propto \frac{1}{R} \\
T \propto \bar{E} \propto \frac{1}{R} .
\end{gathered}
$$

The number of photons does not change as the universe expands. The number of photons per unit volume $n_{\gamma}$ decreases as the volume expands so

$$
n_{\gamma} \propto \frac{1}{R^{3}} .
$$


The energy per unit volume of the radiation is

$$
\rho_{\gamma}=n_{\gamma} \bar{E}
$$

This explains Eq. (4.6). For radiation in equilibrium at temperature $T$, the StefanBoltzmann-Planck formulas give ${ }^{6}$

$$
n_{\gamma} \propto T^{3}, \quad \rho_{\gamma} \propto T^{4}
$$

which agree with Eqs. (4.6), (4.10) and (4.11). It all fits together.

The energy density of vacuum does not change:

$$
\frac{d}{d t} \rho_{v}=0
$$

That means

$$
\frac{d}{d t} \rho_{v} R^{3}=\rho_{v} \frac{d}{d t} R^{3}
$$

so Eq. (4.2) implies

$$
p_{v}=-\rho_{v} .
$$

There are layers of understanding this. Superficially we can say the energy density of vacuum does not change because there is nothing there to change. To probe more deeply, we remember that the vacuum is supposed to be Lorentz invariant. In a homogeneous universe, the energy density is constant throughout space. The spatial gradient of the energy density is zero. Lorentz transformations mix space and time derivatives, so for Lorentz invariance the time derivative of the energy density must be zero too. We can explore this further by considering the energy-momentum tensor. It involves both energy density and pressure. Its Lorentz invariance requires that they are related by Eq. (4.16). That is shown in Appendix B.

Vacuum energy and pressure have the same effect as a cosmological constant in the equations of general relativity. Einstein used the symbol $\Lambda$ for the cosmological constant and it is still often used to label vacuum energy and pressure.

Problem 4.1. Suppose there is an energy density $\rho_{w}$ and pressure $p_{w}$ related by

$$
p_{w}=w \rho_{w}
$$

with $w$ a constant. Show that $\rho_{w} R^{3+3 w}$ stays constant as the universe expands so the time dependence of $\rho_{w}$ is determined by

$$
\rho_{w} \propto R^{-3-3 w}
$$

Note that Eqs. (4.4), (4.6) and (4.14) are obtained as special cases when $w$ is $0,1 / 3$ and -1. A variety of hypotheses about contents of the universe ${ }^{7}$ is covered by broad use of the name "quintessence" and a pressure/energy ratio $w$, particularly for $w$ less than $-1 / 3$, but also more generally for time-dependent as well as constant $w$. 


\section{TIME DEPENDENCE}

Many features of cosmology can be explained with calculations using the equations we have developed. A few central examples are worked out here. The equations yield solutions that describe both expansion and contraction of the universe. We will focus on expansion.

As the universe expands, the energy density of radiation decreases faster, Eq. (4.6), than that of matter, Eq. (4.4), and the energy density of vacuum does not decrease at all, Eq. (4.14). At early times, the energy density of radiation must have been dominant. If there is vacuum energy density, there will be a time in the future when it is dominant. In our universe, it appears that for a time in between, the energy density of matter is important if not dominant.

The time dependence of the expansion of the universe is different for the pressures of radiation, matter and vacuum, so it is different in eras when different kinds of energy are dominant. To see the simplest examples, suppose $k$ is zero or is negligible in the Friedmann equation (as it is at early times when the term involving $k$ is overwhelmed by that involving the energy density of radiation). Then the Friedmann equation says

$$
\left(\frac{d R}{d t}\right)^{2} \propto \rho R^{2}
$$

We consider simple cases where the universe contains only one constituent, either radiation, matter, or vacuum. For radiation, from Eq. (4.6), we see that

$$
\frac{d R}{d t} \propto \frac{1}{R}
$$

So

$$
R \propto t^{\frac{1}{2}}
$$

For matter, from Eq. (4.4), we get

$$
\begin{gathered}
\frac{d R}{d t} \propto \frac{1}{R^{\frac{1}{2}}} \\
R \propto t^{\frac{2}{3}} .
\end{gathered}
$$

For vacuum, from Eq. (4.14), we get

$$
\begin{aligned}
& \frac{d R}{d t} \propto R \\
& R \propto e^{H t}
\end{aligned}
$$

with constant $H=(d R / d t) / R$. This exponential expansion produced by vacuum energy is called inflation. If radiation, matter and vacuum all contribute, the density and pressure are sums

$$
\begin{gathered}
\rho=\rho_{\gamma}+\rho_{m}+\rho_{v} \\
p=p_{\gamma}+p_{v}
\end{gathered}
$$

and the time dependence is more complicated. 
Problem 5.1. Calculate the Hubble parameter $H$ as a function of time for the two cases described by Eqs. (5.3) and (5.5) where $k$ is zero and the density is either only from radiation or only from matter.

Problem 5.2. Show that if $k$ is 0 and the energy and pressure are as described in Problem 4.1 for a constant $w$, then

$$
R \propto t^{\frac{2}{3+3 w}}
$$

Note that Eqs. (5.3) and (5.5) are obtained as special cases when $w$ is $1 / 3$ and 0.

Problem 5.3. Show that if $k$ is 0 and the energy density is all from matter and vacuum, with $\rho_{m}$ and $\rho_{v}$ both positive, then ${ }^{8}$

$$
R^{\frac{3}{2}} \propto \sinh \sqrt{6 \pi G \rho_{v}} t
$$

\section{CRITICAL DENSITY}

When $k$ is zero, the Friedmann equation (2.5) says the density is

$$
\rho=\frac{3 H^{2}}{8 \pi G}
$$

This is called the critical density. Let

$$
\Omega=\frac{\rho}{3 H^{2} / 8 \pi G}
$$

Then $\Omega$ is 1 if $k$ is zero. From the Friedmann equation we see $\Omega$ is less than 1 if $k$ is -1 and $\Omega$ is larger than 1 if $k$ is 1 . Also let

$$
\begin{aligned}
& \Omega_{\gamma}=\frac{\rho_{\gamma}}{\rho} \Omega \\
& \Omega_{m}=\frac{\rho_{m}}{\rho} \Omega \\
& \Omega_{v}=\frac{\rho_{v}}{\rho} \Omega .
\end{aligned}
$$

We can write the Friedmann equation (2.5) as

$$
\frac{\rho-3 H^{2} / 8 \pi G}{\rho}=\frac{3 k R\left(t_{1}\right)^{2}}{8 \pi G \rho R^{2}} .
$$

The left side is the fraction of the energy density that is different from the critical density. We know from observations that right now in our universe this fraction is small. From the right side of the equation we can see how this fraction changes as a function of $R$ as the universe expands. In particular, using Eq. (4.6), we see that in the early universe when the 
energy density was mostly from radiation, the fraction (6.4) increased in proportion to $R^{2}$. It grew very much larger as the universe expanded. Since it is small now, it must have been very small at early times. That begs for explanation. The standard explanation is that in the very early universe there was a period of inflationary expansion when the energy density was mostly from vacuum. Then, since vacuum energy density stays constant, the fraction (6.4) decreased in proportion to $1 / R^{2}$. It became very very small as $R$ grew exponentially, as described by Eq. (5.7).

Problem 6.1. Show that if the value of $\Omega$ is 1 at a particular time, then it is constant in time.

Problem 6.2. Can $\Omega_{v}$ be constant in time? What is required for that to happen?

\section{ACCELERATION}

For the present and future expansion of the universe we consider only matter and vacuum. The energy density of radiation, which was so important in the early universe, is negligible now.

The acceleration of the expansion of the universe is described by Eq. (3.4). With only $\rho_{m}$ and $\rho_{v}$ included, it is

$$
\frac{d^{2} R}{d t^{2}}=-\frac{4}{3} \pi G\left(\rho_{m}-2 \rho_{v}\right) R
$$

because $p_{m}$ is zero and $p_{v}$ is $-\rho_{v}$. The acceleration is positive, zero, or negative depending on whether $2 \rho_{v}$ is greater than, equal to, or less than $\rho_{m}$ In the plane of $\Omega_{m}$ and $\Omega_{v}$, the acceleration is zero on the line where $2 \Omega_{v} / \Omega_{m}$ is 1 . For larger $2 \Omega_{v} / \Omega_{m}$ the acceleration is positive and for smaller $2 \Omega_{v} / \Omega_{m}$ the acceleration is negative. (See Fig.1)

Problem 7.1. Suppose the acceleration Eq. (7.1) is zero at a particular time. Can it stay zero in time? What is required for that to happen?

\section{EXPANSION FOREVER?}

Will the expansion of the universe ever stop? That question was easier to discuss a few years ago when only matter density was considered. If $\rho$ is just $\rho_{m}$, we can use our initial Newtonian equations. Both the $M$ in Eqs. (2.2) and (2.3) and the $E$ in Eqs. (2.2) and (2.4) are constant. We can apply these equations in the familiar Newtonian way. Galaxies will stop moving apart, so the expansion will stop, if $E$ is negative. The expansion will not stop if $E$ is zero or positive.

Now we consider energy densities for both matter and vacuum. We want to find the values of $\Omega_{m}$ and $\Omega_{v}$, the area in the plane of $\Omega_{m}$ and $\Omega_{v}$, for which the expansion will stop. We just found that when $\Omega_{v}$ is zero, the expansion will stop if and only if $\Omega_{m}$ is larger than 1 ( because that means $\Omega$ is larger than $1, k$ is positive, and $E$ is negative). The expansion will stop if $d^{2} R / d t^{2}$ is negative and remains negative until $d R / d t$ is zero. We can see this 
happens if $\Omega_{v}$ is negative, because then we see from Eq. (3.4) that

$$
\frac{d^{2} R}{d t^{2}} \propto-\left(\Omega_{m}-2 \Omega_{v}\right)
$$

is negative and will stay negative, and in Eq. (3.3)

$$
\frac{8}{3} \pi G\left(\rho_{m}+\rho_{v}\right) R^{2}
$$

will fall to $k R\left(t_{1}\right)^{2}$ even if $k$ is -1 , making $d R / d t$ zero, as $\rho_{m} R^{2}$ decreases in proportion to $1 / R$ and the magnitude of $\rho_{v} R^{2}$ increases in proportion to $R^{2}$.

Suppose $\Omega_{m} \leq 1$. We have found that the expansion stops if $\Omega_{v}$ is negative but does not stop if $\Omega_{v}$ is zero. We can conclude that it does not stop if $\Omega_{v}$ is positive, because larger $\Omega_{v}$ makes $d^{2} R / d t^{2}$ and $d R / d t$ both larger.

For $\Omega_{m}>1$, there is a similar limit on the $\Omega_{v}$ for which the expansion will stop. The limit values of $\Omega_{v}$ as a function of $\Omega_{m}$ form a curve across the plane of $\Omega_{m}$ and $\Omega_{v}$ (see Fig. 1 ). For smaller $\Omega_{v}$ the expansion will stop and for larger $\Omega_{v}$ it will not, again because larger $\Omega_{v}$ makes $d^{2} R / d t^{2}$ and $d R / d t$ both larger. We know that the expansion stops when $\Omega_{m}$ is larger than 1 and $\Omega_{v}$ is zero, so the limit values of $\Omega_{v}$ are at least zero.

At the limit, $d^{2} R / d t^{2}$ is negative just long enough for $d R / d t$ to reach zero, so $d^{2} R / d t^{2}$ and $d R / d t$ are zero at the same time. We write $\tilde{\rho}_{m}$ and $\tilde{R}$ for the values at that time and $\rho_{m}$ and $R$ for the values now. The value of $\rho_{v}$ is the same at all times. From Eqs. (3.4), (3.3), and (4.4) we get

$$
\begin{gathered}
\tilde{\rho}_{m}-2 \rho_{v}=0 \\
\frac{8}{3} \pi G\left(\tilde{\rho}_{m}+\rho_{v}\right) \tilde{R}^{2}=k R\left(t_{1}\right)^{2}=R\left(t_{1}\right)^{2} \\
\rho_{m} R^{3}=\tilde{\rho}_{m} \tilde{R}^{3}
\end{gathered}
$$

which imply

$$
8 \pi G \rho_{v}\left(\frac{\rho_{m}}{2 \rho_{v}}\right)^{\frac{2}{3}} R^{2}=R\left(t_{1}\right)^{2} .
$$

Using this to substitute for $R_{0}^{2} / R^{2}$ in the Friedmann equation (2.5), where $k$ must be 1 , dividing by $H^{2}$ and using the definitions (6.2) and (6.3) for $\Omega_{m}$ and $\Omega_{v}$, yields

$$
1-\Omega_{m}-\Omega_{v}=-\frac{3}{2^{\frac{2}{3}}} \Omega_{v}^{\frac{1}{3}} \Omega_{m}^{\frac{2}{3}}
$$

which we can write as

$$
4 y^{3}-3 y+x=0
$$

with

$$
x=1-\frac{1}{\Omega_{m}}, \quad y=\left(\frac{\Omega_{v}}{4 \Omega_{m}}\right)^{\frac{1}{3}} .
$$

Let $y=\cos \beta$; that is allowed because $x$ and $y$ are positive and Eq. (8.8) implies $y$ is less than 1 . Using

$$
\cos 3 \beta=4 \cos ^{3} \beta-3 \cos \beta,
$$


and taking $y$ to be zero when $x$ is zero, so $\Omega_{v}$ is zero when $\Omega_{m}$ is 1 , gives

$$
\begin{gathered}
-x=\cos 3 \beta \\
x=\cos (3 \beta-\pi), \quad y=\cos \left(\frac{1}{3} \cos ^{-1} x+\frac{\pi}{3}\right) \\
\Omega_{v}=4 \Omega_{m} \cos ^{3}\left(\frac{1}{3} \cos ^{-1}\left(1-\frac{1}{\Omega_{m}}\right)+\frac{\pi}{3}\right) .
\end{gathered}
$$

On the curve in Fig.1, the values of $\Omega_{v}$ are exaggerated by a factor of 5 to show that they are positive when $\Omega_{m}$ is larger that 1 . Actually they are quite small.

Problem 8.1. For example, show that $\Omega_{v} / \Omega_{m}$ is $1 / 54$ when $\Omega_{m}$ is $54 / 28$ on the curve described by Eq. (8.12), because if $y$ is $1 / 6$ then $x$ is $26 / 54$ in Eq. (8.8).

Problem 8.2. Show that a good approximation to Eq. (8.12), obtained by dropping the first term in Eq. (8.8), is

$$
\Omega_{v}=\frac{4 \Omega_{m}}{27}\left(1-\frac{1}{\Omega_{m}}\right)^{3}
$$

\section{TRAVEL TIME}

We can calculate the relation between the redshift of light from a distant galaxy and the time the light takes to reach us. It depends on the density of matter and vacuum energy.

For light that was emitted from a distant galaxy at wavelength $\lambda$ and is observed here now at wavelength $\lambda_{0}$ the redshift parameter $z$ is defined by

$$
1+z=\frac{\lambda_{0}}{\lambda}
$$

As the universe expands, the wavelengths of light expand in proportion to distances. Let

$$
r=\frac{R}{R_{0}}
$$

with $R$ and $r$ functions of time and $R_{0}$ the value of $R$ now. Then at the time the light was emitted

$$
r=\frac{1}{1+z} .
$$

It is handy to work with $r$ instead of $R$ and measure time in units of $1 / H_{0}$ where $H_{0}$ is the value of $H$ now. Let

$$
\tau=H_{0} t,
$$

so $\tau$ is 1 when $t$ is $1 / H_{0}$. Using Eq. (4.4), we can write

$$
\rho_{m}=\rho_{m 0}\left(\frac{R_{0}}{R}\right)^{3}=\frac{\rho_{m 0}}{r^{3}}
$$


where $\rho_{m 0}$ is the value of $\rho_{m}$ now. Then using this, Eqs. (4.14), (6.2) and (6.3), we can write Eq. (7.1) as

$$
\frac{d^{2} r}{d \tau^{2}}=-\frac{1}{2} \frac{\Omega_{m 0}}{r^{2}}+\Omega_{v 0} r
$$

where $\Omega_{m 0}$ and $\Omega_{v 0}$ are the values of $\Omega_{m}$ and $\Omega_{v}$ now. From the Hubble law now

$$
\frac{d R}{d t}=H_{0} R
$$

we get

$$
\frac{d r}{d \tau}=r=1
$$

at $r=1$. Remembering that Eq. (7.1) is a version of Eq. (3.4) which was obtained by taking the time derivative of Eq. (3.3), we can deduce, and/or check by differentiating again, that

$$
\left(\frac{d r}{d \tau}\right)^{2}=\frac{\Omega_{m 0}}{r}+\Omega_{v 0} r^{2}+\text { constant }
$$

Assuming that $d r / d \tau$ is positive, and choosing the constant to get the value (9.8) when $r$ is 1 , we obtain

$$
\frac{d \tau}{d r}=\left(\frac{\Omega_{m 0}}{r}+\Omega_{v 0} r^{2}+1-\Omega_{m 0}-\Omega_{v 0}\right)^{-\frac{1}{2}}
$$

We have not needed to know or assume anything about the constant term in the Friedmann equation(3.3).

Integrating gives time, in units of $1 / H_{0}$, as a function of the distance fraction $r$ or redshift $z$. For each $\Omega_{m 0}, \Omega_{v 0}$, the time the light takes to reach us is

$$
(\Delta \tau)(r)=\int_{r}^{1} \frac{d \tau}{d r}\left(r^{\prime}\right) d r^{\prime}
$$

with $d \tau / d r$ given by Eq. (9.10). We can see qualitatively how this time depends on $\Omega_{m 0}$ and $\Omega_{v 0}$. Since $r$ in the integral is less than 1, the $\Omega_{m 0} / r$ in Eq. (9.10) is larger than the $\Omega_{m 0}$ and $\Omega_{v 0} r^{2}$ is smaller than $\Omega_{v 0}$, so larger $\Omega_{m 0}$ gives smaller time and larger $\Omega_{v 0}$ gives larger time. ${ }^{9}$

The integrand (9.10) for $(\Delta \tau)(r)$ is valid as long as matter and vacuum provide the dominant forms of energy density. Radiation becomes important for $z$ larger than about 10,000. If there are no other forms of energy density, extending the integral $(9.11)$ to $(\Delta \tau)(0)$ gives a very good approximation to the age of the universe for each $\Omega_{m 0}$ and $\Omega_{v 0}$.

Einstein's cosmological constant, which we now interpret as vacuum energy, was introduced originally to get equations that can describe a static universe. That can not be described by the equations we are using here. In particular, Eq. (9.8) is clearly inconsistent with a static universe; the assumption we need to make to use it is that the universe is expanding. The way we are doing things here leaves a piece of history behind.

Problem 9.1. Show that if there is only vacuum energy density and $\Omega_{v 0}$ is positive and less than 1 , then

$$
r=\left(\frac{1-\Omega_{v 0}}{\Omega_{v 0}}\right)^{\frac{1}{2}} \sinh \Omega_{v 0}^{\frac{1}{2}} \tau
$$


Notice that $r \rightarrow \tau$ as $\Omega_{v 0}$ approaches zero, and the age of the universe becomes infinite as $\Omega_{v 0}$ approaches 1 .

Problem 9.2. Find how $r$ varies as a function of $\tau$ when there is only vacuum energy density and $\Omega_{v 0}$ is 1 . Show that then the age of the universe is infinite.

Problem 9.3. Show that if there is only vacuum energy density and $\Omega_{v 0}$ is larger than 1 , then there is no big bang because $d r / d \tau$ does not stay positive for $r$ between zero and 1 .

Problem 9.4. Suppose there is only matter and vacuum energy density. By looking at the minimum of $(d r / d \tau)^{2}$ as a function of $\mathrm{r}$, show that there is no big bang, because $d r / d \tau$ does not stay positive for $\mathrm{r}$ between zero and 1 , if

$$
\left(2^{\frac{1}{3}}+2^{-\frac{2}{3}}\right) \Omega_{m 0}^{\frac{2}{3}} \Omega_{v 0}^{\frac{1}{3}}+1-\Omega_{m 0}-\Omega_{v 0}
$$

is not positive when $\Omega_{m 0}$ is less than $2 \Omega_{v 0}$. Sketch the curve that bounds the area in the $\Omega_{m 0}, \Omega_{v 0}$ plane where this happens. Show that the age of the universe grows infinitely large as this area is approched. ${ }^{\mathbf{9}}$

Problem 9.5. Suppose there is only matter density and $\Omega_{m 0}$ is 1 . Calculate $(\Delta \tau)(r)$ and the age of the universe $(\Delta \tau)(0)$. Expand $(\Delta \tau)\left((1+z)^{-1}\right)$ as a series in powers of $z$ to second order, which can be used for small $z$ for $r$ near 1 .

Problem 9.6. By changing the variable from $r$ to $z$ in the integral (9.11) and expanding in powers of $z$, show that to second order in $z$, for small $z$ for $r$ near 1 ,

$$
(\Delta \tau)\left((1+z)^{-1}\right)=z-z^{2}-\frac{1}{4}\left(\Omega_{m 0}-2 \Omega_{v 0}\right) z^{2}
$$

Problem 9.7. Sketch the age of the universe $(\Delta \tau)(0)$ as a function of $\Omega_{m 0}$ for $\Omega_{m 0}$ between 0 and 1 for the cases where:

(a) there is only matter energy density; and

(b) there is both matter and vacuum, $\Omega_{m 0}$ and $\Omega_{v 0}$ are both positive, and their sum is 1 .

The relative positions of the curves and the directions of their slopes can be deduced from the observations made following equation Eq. (9.11). The values when $\Omega_{m 0}$ is 1 are found in Problem 9.5. For (b), the behavior as $\Omega_{m 0}$ approaces 0 is found in Problems 9.1 and 9.2. For (a), the value when $\Omega_{m 0}$ is 0 can be deduced by seeing that $d \tau / d r$ is 1 .

Problem 9.8. Suppose there is only radiation energy density and $\Omega_{\gamma 0}$, the value of $\Omega_{\gamma}$ now, is 1. ¿From Eq. (5.3) and the requirement that $d \tau / d r$ is 1 when $r$ is 1 deduce that

$$
(\Delta \tau)(r)=\frac{1}{2}\left(1-r^{2}\right)
$$

In particular, the age of the universe in units of $1 / H_{0}$ is

$$
(\Delta \tau)(0)=\frac{1}{2} .
$$


Problem 9.9. Show that if there were only radiation energy density, Eq. (9.10) would be replaced by

$$
\frac{d \tau}{d r}=\left(\frac{\Omega_{\gamma 0}}{r^{2}}+1-\Omega_{\gamma 0}\right)^{-\frac{1}{2}}
$$

with $\Omega_{\gamma 0}>0$ the value of $\Omega_{\gamma}$ now. Show that this gives times $(\Delta \tau)(r)$ that are smaller for larger $\Omega_{\gamma 0}$ and for each $\Omega_{\gamma 0}$ are smaller than they would be if there were only matter energy density with $\Omega_{m 0}$ the same as $\Omega_{\gamma 0}$. Sketch the age of the universe $(\Delta \tau)(0)$ as a function of $\Omega_{\gamma 0}$ for $\Omega_{\gamma 0}$ between 0 and 1. (Find the value when $\Omega_{\gamma 0}$ is 0 by seeing that $d \tau / d r$ is 1 and use the result of Problem 9.8 for the value when $\Omega_{\gamma 0}$ is 1.) Compare this sketch with the sketches made in Problem 9.7. Show, by calculating the derivative with respect to $r$ and checking the value when $r$ is 1 , that

$$
(\Delta \tau)(r)=\frac{1-\sqrt{\Omega_{\gamma 0}+\left(1-\Omega_{\gamma 0}\right) r^{2}}}{1-\Omega_{\gamma 0}} .
$$

Show that in particular

$$
(\Delta \tau)(0)=\frac{1}{1+\sqrt{\Omega_{\gamma 0}}}
$$

and check that this agrees with your sketch.

Problem 9.10. Suppose the energy and pressure are as described in Problems 4.1 and 5.2 with constant $w$, but $w$ not -1 , and $\Omega_{w 0}$, the present ratio of this " $w$ " energy density to the critical density, is 1. From the result of Problem 5.2 and the requirement that $d \tau / d r$ is 1 when $r$ is 1 deduce that

$$
(\Delta \tau)(r)=\frac{2}{3+3 w}\left(1-r^{\frac{3+3 w}{2}}\right) .
$$

In particular, the age of the universe in units of $1 / H_{0}$ is

$$
(\Delta \tau)(0)=\frac{2}{3+3 w} .
$$

Check that you get the result of Problem 9.5 when $w$ is 0 and of Problem 9.8 when $w$ is $1 / 3$.

Problem 9.11. Show that if the vacuum energy and pressure are replaced by the $w$ energy and pressure described in Problems 4.1, 5.2 and 9.10, for a constant $w$, then Eq. (9.10) is replaced by

$$
\frac{d \tau}{d r}=\left(\Omega_{m 0} r^{-1}+\Omega_{w 0} r^{-1-3 w}+1-\Omega_{m 0}-\Omega_{w 0}\right)^{-\frac{1}{2}}
$$

where $\Omega_{w 0}$ is the present ratio of the $w$ energy density to the critical density. Note that when $w$ is $-1 / 3$ this and the travel times $(\Delta \tau)(r)$ are the same as for matter alone. Check that you get the expected results when $w$ is 0 or -1 . Show that if $w$ is less than $-1 / 3$, the times $(\Delta \tau)(r)$ are larger for larger $\Omega_{w 0}$; in particular, they are larger when $\Omega_{w 0}$ is positive than when $\Omega_{w 0}$ is 0 . Suppose $\Omega_{m 0}$ and $\Omega_{w 0}$ are both positive, their sum is 1 , and $w$ is $-1 / 2$. 
Sketch the age of the universe $(\Delta \tau)(0)$ as a function of $\Omega_{m 0}$ for $\Omega_{m 0}$ between 0 and 1 , as in Problem 9.7, taking the value when $\Omega_{m 0}$ is 1 from Problem 9.5 and the value when $\Omega_{m 0}$ is 0 from Problem 9.10. By calculating the derivative with respect to $r$ and checking the value when $r$ is 1 , show that

$$
(\Delta \tau)(r)=\frac{4}{3 \Omega_{w 0}}\left(1-\sqrt{\Omega_{m 0}+\Omega_{w 0} r^{\frac{3}{2}}}\right) .
$$

Show that in particular

$$
(\Delta \tau)(0)=\frac{4}{3\left(1+\sqrt{\Omega_{m 0}}\right)} .
$$

Check that this agrees with your sketch and compare it with the result of Problem 9.9 for radiation.

Problem 9.12. By calculating the derivative with respect to $r$ and checking the value when $r$ is 1 , show that if $\Omega_{m 0}$ and $\Omega_{v 0}$ are both positive and their sum is 1 , then ${ }^{\mathbf{1 0}}$

$$
(\Delta \tau)(r)=\frac{2}{3} \frac{1}{\sqrt{\Omega_{v 0}}}\left[\sinh ^{-1} \sqrt{\frac{\Omega_{v 0}}{\Omega_{m 0}}}-\sinh ^{-1} \sqrt{\frac{\Omega_{v 0}}{\Omega_{m 0}}} r^{\frac{3}{2}}\right] .
$$

In particular, the age of the universe in units of $1 / H_{0}$ as a function of $\Omega_{m 0}$ for $\Omega_{m 0}$ between 0 and 1 is

$$
(\Delta \tau)(0)=\frac{2}{3}\left(1-\Omega_{m 0}\right)^{-\frac{1}{2}} \sinh ^{-1}\left(\frac{1}{\Omega_{m 0}}-1\right)^{\frac{1}{2}} .
$$

Check that this agrees with the sketch you made for Problem 9.7(b).

Problem 9.13. Suppose there is only matter density and $\Omega_{m 0}$ is positive and less than 1 . By calculating the derivative with respect to $r$ and checking the value when $r$ is 1 , show that $^{11}$

$$
\begin{aligned}
& (\Delta \tau)(r)=\frac{1-r \sqrt{\Omega_{m 0} / r+1-\Omega_{m 0}}}{1-\Omega_{m 0}} \\
& +\frac{\Omega_{m 0}}{2\left(1-\Omega_{m 0}\right)^{\frac{3}{2}}}\left[\cosh ^{-1}\left(1+2 \frac{1-\Omega_{m 0}}{\Omega_{m 0}} r\right)-\cosh ^{-1}\left(1+2 \frac{1-\Omega_{m 0}}{\Omega_{m 0}}\right)\right] .
\end{aligned}
$$

In particular, the age of the universe in units of $1 / H_{0}$ as a function of $\Omega_{m 0}$ for $\Omega_{m 0}$ between 0 and 1 is

$$
(\Delta \tau)(0)=\frac{1}{1-\Omega_{m 0}}-\frac{\Omega_{m 0}}{2\left(1-\Omega_{m 0}\right)^{\frac{3}{2}}} \cosh ^{-1}\left(1+2 \frac{1-\Omega_{m 0}}{\Omega_{m 0}}\right) .
$$

Check that this agrees with the sketch you made for Problem 9.7(a).

\section{HUBBLE PLOTS}

Hubble plots are providing a more detailed picture of the universe as they extend to larger distances. Earlier plots showed galaxies receding from us with velocities proportional to their distances, as expected in a uniformly expanding universe. Now Hubble plots are showing how the expansion has developed during the time light from distant galaxies has been traveling to us. ${ }^{\mathbf{1 2}}$ 
Recession velocities are measured from red shifts of spectral lines. Hubble plots now show distance as a function of red shift. Velocity is considered to be a secondary quantity that can be calculated from the formula for the relativistic Doppler effect.

The distance to an object is found by comparing its apparent luminosity, how bright it looks, with its absolute luminosity, how bright it is thought to actually be. Apparent luminosity decreases with distance, but the expansion of the distance scale, the curvature of space, and the redshift all combine to make the distance used to describe luminosity different from the speed of light times the travel time.

If the redshift is $z$, the light from the object is spread over a sphere of radius

$$
\chi\left(r=(1+z)^{-1}\right)=\int_{r}^{1} \frac{1}{r^{\prime}} \frac{d \tau}{d r^{\prime}}\left(r^{\prime}\right) d r^{\prime}
$$

in units of $1 / H_{0}$, because distances traveled at earlier times are expanded by factors of $1 / r^{\prime}$. If space is flat, has no curvature, the light is spread over a sphere of area $4 \pi\left(\chi / H_{0}\right)^{2}$. General relativity says space may be curved, so the area of the sphere of light is $4 \pi\left(\sigma / H_{0}\right)^{2}$ where $\sigma$ is $\chi$ if there is no curvature, but

$$
\sigma=r_{c} \sin \left(\frac{\chi}{r_{c}}\right)
$$

for positive curvature, and

$$
\sigma=r_{c} \sinh \left(\frac{\chi}{r_{c}}\right)
$$

for negative curvature, with

$$
r_{c}=\left|1-\Omega_{0}\right|^{-\frac{1}{2}}
$$

where $\Omega_{0}=\Omega_{m 0}+\Omega_{v 0}$ is the value of $\Omega$ now. ${ }^{\mathbf{1 3}}$ The two-dimensional analog that is easy to picture is that on the two-dimensional surface of a sphere of radius $r_{c}$ the circumference of a circle of radius $\chi$ is $2 \pi r_{c} \sin \left(\chi / r_{c}\right)$.

The redshift decreases the frequencies of the light and the energies of the photons by a factor of $(1+z)^{-1}$, and the frequencies of arrival of photons, the numbers of photons arriving per unit time, are decreased by the same factor, so the redshift decreases the intensity of the light by a factor of $(1+z)^{-2}$. The "luminosity distance" $d_{L}$ that is used in Hubble plots is defined by

$$
d_{L}=(1+z) \frac{\sigma}{H_{0}}
$$

so that

$$
\frac{1}{4 \pi d_{L}^{2}}=\frac{1}{(1+z)^{2} 4 \pi\left(\sigma / H_{0}\right)^{2}} .
$$

It is what we would deduce the distance to the object to be if the relation between its apparent luminosity and absolute luminosity came just from the light spreading out over a sphere in flat space. If there is only matter density, the integral (10.1) with Eq. (9.10) for $d \tau / d r$ yields the simple result that ${ }^{\mathbf{1 4}}$

$$
d_{L}=\frac{2}{H_{0} \Omega_{m 0}^{2}}\left[\Omega_{m 0} z+\left(2-\Omega_{m 0}\right)\left(1-\sqrt{\Omega_{m 0} z+1}\right)\right]
$$

for all three cases of positive, zero, or negative curvature where $\Omega_{m 0}$ is greater than, equal to, or less than 1. 
Problem 10.1. Show that Eq. (10.7) holds when there is only matter density, no curvature, and $\Omega_{m 0}$ is 1 .

Problem 10.2. Suppose there is only matter density and $\Omega_{m 0}$ is larger than 1. By calculating the derivative with respect to $r$ and checking the value when $r$ is 1 , show that

$$
\chi(r)=\frac{2}{\sqrt{\Omega_{m 0}-1}}\left[\sin ^{-1} \sqrt{\frac{\Omega_{m 0}-1}{\Omega_{m 0}}}-\sin ^{-1} \sqrt{\frac{\Omega_{m 0}-1}{\Omega_{m 0}} r}\right] .
$$

Show that this and Eqs. (10.2), (10.4) and (10.5) give the result (10.7) for the luminosity distance $d_{L}$. (The case where $\Omega_{m 0}$ is less than 1 is covered in Problem 10.9.)

Problem 10.3. By changing the variable from $r$ to $z$ in the integral (10.1), using Eq. (9.10) for $d \tau / d r$, and expanding in powers of $z$, show that to second order in $z$, for small $z$ for $r$ near 1 ,

$$
H_{0} d_{L}=z+\frac{1}{2} z^{2}-\frac{1}{4}\left(\Omega_{m 0}-2 \Omega_{v 0}\right) z^{2}
$$

for all three cases of positive, zero, or negative curvature for any $\Omega_{m 0}$ and $\Omega_{v 0}$.

Problem 10.4. Suppose $\Omega_{m 0}$ and $\Omega_{v 0}$ are both positive and their sum is 1 . By making observations like those made for travel times following Eq. (9.11), show that for each $z$ the luminosity distance $d_{L}$ is smaller for larger $\Omega_{m 0}$ and smaller $\Omega_{v 0}$.

Problem 10.5. Suppose there is only matter with $\Omega_{m 0}$ positive and less than 1 . Show, using Eqs. (9.10), (10.1) and (10.3)-(10.5), that for each $z$ the luminosity distance $d_{L}$ is smaller for larger $\Omega_{m 0}$.

Problem 10.6. Suppose vacuum is replaced by $w$ energy and pressure as in Problem 9.11. Suppose $\Omega_{m 0}$ and $\Omega_{w 0}$ are both positive and their sum is 1. Show that for each $z$ and fixed $\Omega_{m 0}$ the luminosity distance $d_{L}$ is smaller for larger $w$, and for each $z$ and fixed negative $w$, the luminosity distance $d_{L}$ is smaller for larger $\Omega_{m 0}$.

Problem 10.7. Suppose there is only $w$ energy and pressure as described in Problems 4.1, 5.2, 9.10 and 9.11, and suppose $\Omega_{w 0}$ is 1 . Show that if $w$ is not $-1 / 3$ then

$$
H_{0} d_{L}=\frac{2}{3 w+1}(1+z)\left[1-(1+z)^{-\frac{3 w+1}{2}}\right]
$$

Problem 10.8. Suppose vacuum is replaced by $w$ energy and pressure as in Problems 9.11 and 10.6. Now let $w$ be $-1 / 3$. Suppose $\Omega_{m 0}$ and $\Omega_{w 0}$ are both positive and their sum is 1. Then the luminosity distance is

$$
d_{L}=(1+z) \frac{\chi(r)}{H_{0}}
$$


with $\chi(r)$ given by Eqs. (10.1) and (9.10). Show, by calculating the derivative with respect to $r$ and checking the value when $r$ is 1 , that

$$
\chi(r)=\frac{2}{\sqrt{1-\Omega_{m 0}}}\left[\sinh ^{-1} \sqrt{\frac{1-\Omega_{m 0}}{\Omega_{m 0}}}-\sinh ^{-1} \sqrt{\frac{1-\Omega_{m 0}}{\Omega_{m 0}} r}\right] .
$$

Observe that $\chi(r)$ would be the same if there were only matter, with the same $\Omega_{m 0}$, but show, using Eq. (10.3), that the luminosity distance $d_{L}$ would be larger.

Problem 10.9. Suppose there is only matter, with $\Omega_{m 0}$ positive and less than 1 . Show that Eqs. (10.3) - (10.5) and the result of Problem 10.8 for $\chi(r)$ give the formula (10.7) for the luminosity distance $d_{L}$. 


\section{APPENDIX A. RADIATION PRESSURE}

Consider the pressure on a small plane area $A$ from photons of momentum $\vec{p}$ reflecting off it. Let $p_{x}$ be the component of $\vec{p}$ perpendicular to $A$

for a photon approaching $A$. Reflection changes $p_{x}$ to $-p_{x}$ and leaves the other components of $\vec{p}$ unchanged. Force is the rate of change of momentum, so the force on $A$ is $2 p_{x}$ times the number of photons that hit $A$ per unit time. Altogether, including photons with different momenta $\vec{p}$, the force on $A$ is

$$
\frac{1}{2} 2 \overline{p_{x} v_{x}} A n_{\gamma}
$$

where $v_{x}$ is the $x$ component of the velocity of a photon with momentum $\vec{p}$ and $n_{\gamma}$ is the number of photons per unit volume. The average is over all $\vec{p}$, but only photons with positive $p_{x}$ exert force on $A$. They are just half of the photons present. That explains the factor $1 / 2$. The velocity of a photon is $c$ in the direction of its momentum. We assume there is a uniform distribution of momentum directions. Then the pressure is

$$
\begin{gathered}
p_{\gamma}=\overline{p_{x} v_{x}} n_{\gamma}=\overline{p_{x}\left(p_{x} /|\vec{p}|\right)} c n_{\gamma}=c \frac{1}{3} \frac{\overline{p_{x}^{2}+p_{y}^{2}+p_{z}^{2}}}{|\vec{p}|} n_{\gamma} \\
=\frac{1}{3} c \overline{|\vec{p}|} n_{\gamma}=\frac{1}{3} \rho_{\gamma}
\end{gathered}
$$

because the average does not depend on the direction of the momentum and the energy of a photon is

$$
h \nu=c \frac{h}{\lambda}=c|\vec{p}|
$$

\section{APPENDIX B. VACUUM PRESSURE}

The energy density is the time-time component $T_{00}$ of a relativistic tensor $T_{\mu \nu}$. That tells us how it is changed by Lorentz transformations. It is more complicated than the density of something that is Lorentz invariant, for example electric charge. If an observer sees electric charges at rest occupying a volume $V$ with charge density $\rho_{e}$, an observer who sees the charges moving with velocity $\vec{v}$ will see the same total charge $\rho_{e} V$ occupying volume $V \sqrt{1-\vec{v}^{2}}$ with density $\rho_{e} / \sqrt{1-\vec{v}^{2}}$. Charge density is the time component $j_{0}$ of a relativistic four-vector

$$
j_{\mu}=\left(\frac{\rho_{e}}{\sqrt{1-\vec{v}^{2}}}, \frac{\rho_{e} \vec{v}}{\sqrt{1-\vec{v}^{2}}}\right) .
$$

The space part $\vec{j}$ is charge per unit volume times velocity. It is the current density. It gives the charge crossing unit area per unit time.

The energy of an object is not Lorentz invariant. If an observer sees the object at rest with energy $m$, an observer who sees it moving with velocity $\vec{v}$ will say it has energy $m / \sqrt{1-\vec{v}^{2}}$. Its energy is the time component $p_{0}$ of a relativistic four-vector

$$
p_{\mu}=\left(\frac{m}{\sqrt{1-\vec{v}^{2}}}, \frac{m \vec{v}}{\sqrt{1-\vec{v}^{2}}}\right) .
$$


The space part $\vec{p}$ is the object's momentum. Energy density is the time-time component $T_{00}$ of a relativistic tensor $T_{\mu \nu}$ like $p_{\mu} p_{\nu}$. The other $T_{0 \nu}$ components are momentum density or energy current. The space-space components $T_{j k}$, where $j$ and $k$ are 1,2 , or 3 , are momentum current. They give momentum per unit time crossing unit area. That is pressure.

In a homogeneous uniformly expanding universe, for observers who see the nearby universe at rest, $T_{\mu \nu}$ must be zero for $\mu \neq \nu$. There is no momentum or flow of energy, and no shear pressure (for example, no flow in the $\mathrm{x}$ direction of momentum in the $\mathrm{y}$ direction). Also

$$
T_{11}=T_{22}=T_{33} ;
$$

if not, a coordinate rotation would make $T_{j k}$ nonzero for some $j$ different from $k$. So

$$
T_{\mu \nu}=\left(\begin{array}{cccc}
\rho & 0 & 0 & 0 \\
0 & p & 0 & 0 \\
0 & 0 & p & 0 \\
0 & 0 & 0 & p
\end{array}\right) .
$$

In a homogeneous universe, $\rho$ and $p$ must be the same throughout space. They can be functions only of time.

A Lorentz transformation changes $T_{\mu \nu}$ to

$$
T_{\mu \nu}^{\prime}=\sum_{\alpha=0}^{3} \sum_{\beta=0}^{3} \Lambda_{\mu \alpha} \Lambda_{\nu \beta} T_{\alpha \beta}
$$

where $\Lambda$ is the $4 \times 4$ matrix for the Lorentz transformation of space-time coordinates. For an observer moving with velocity $-v$ in the $z$ direction, for example,

$$
\Lambda=\left(\begin{array}{cccc}
\frac{1}{\sqrt{1-v^{2}}} & 0 & 0 & \frac{v}{\sqrt{1-v^{2}}} \\
0 & 1 & 0 & 0 \\
0 & 0 & 1 & 0 \\
\frac{v}{\sqrt{1-v^{2}}} & 0 & 0 & \frac{1}{\sqrt{1-v^{2}}}
\end{array}\right)
$$

gives

$$
\begin{aligned}
& T_{00}^{\prime}=\frac{\rho+v^{2} p}{1-v^{2}} \\
& T_{33}^{\prime}=\frac{p+v^{2} \rho}{1-v^{2}} .
\end{aligned}
$$

The energy-momentum tensor is Lorentz invariant, $T_{\mu \nu}^{\prime}$ is the same as $T_{\mu \nu}$, if and only if

$$
p=-\rho .
$$

If the vacuum is Lorentz invariant, then $p_{v}$ is $-\rho_{v}$. 


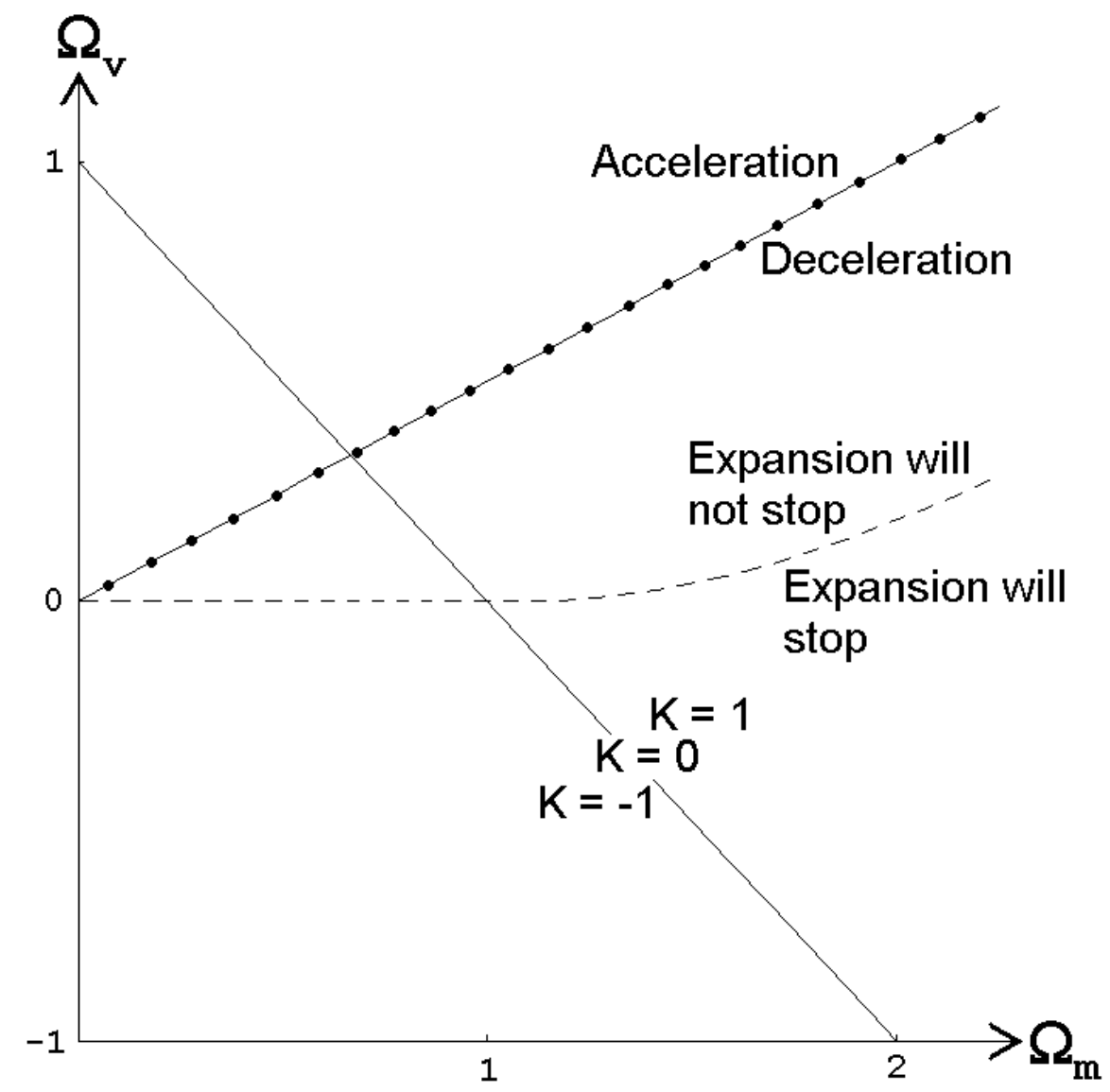

Figure 1. Dependence of cosmology on the densities of matter and vacuum energies. For densities on the solid line, $\mathrm{k}$ is 0 . Above the solid line, $\mathrm{k}$ is 1 ; below it is -1 . For densities on and above the dashed curve, the expansion of the universe will not stop; below it will stop. For densities on the dotted line, the expansion of the universe has no acceleration. Above the dotted line, the expansion of the universe accelerates; below it decelerates.

[1] E. A. Milne, "A Newtonian expanding universe", Quart. J. Math. (Oxford) 5, 64-72 (1934); W. H. McCrea and E. A. Milne, "Newtonian universes and the curvature of space", Quart. J. Math. (Oxford) 5, 73-80 (1934); H. Bondi, Cosmology (Cambridge U. P., Cambridge, 1960), Chapter IX; C. Callan, R. H. Dicke, and P. J. E. Peebles, "Cosmology and Newtonian mechanics", Am. J. Phys. 33, 105-8 (1965); D. W. Sciama, Modern Cosmology (Cambridge U. P., Cambridge, 1971), Chapter 8; S. Weinberg, Gravitation and cosmology: principles and applications of the general theory of relativity (Wiley, New York, 1972), pp. 474-5; J. Silk, 
The Big Bang, Third Edition (Freeman, New York, 2001), Mathematical Note 5; J. Rich, Fundamentals of Cosmology (Springer - Verlag, Berlin, 2001), pp. 19-21. Weinberg and Silk derive the Friedmann equation directly from energy conservation. The others use Newtonian gravitational force, which yields equations that are valid only for zero pressure and have to be changed when there is pressure.

[2] D. Goldsmith, The runaway universe (Perseus, Cambridge, MA, 2000); J. P. Ostriker and P. J. Steinhardt, "The quintessential universe", Scientific American 284, Number 1, 46-53 (January 2001).

[3] S. Weinberg, reference 1, Chapter 14, Section 6, and The First Three Minutes (Basic Books, New York, 1977), Chapter II; J. Silk, reference 1, Chapter 5; D. Goldsmith, reference 2, Chapter 4.

[4] S. Wienberg, The First Three Minutes, reference 3, Chapter III; J. Silk, reference 1, Chapter 3; D. Goldsmith, reference 2, Chapter 11.

[5] S. Wienberg, reference 1, Chapter 15, Section 1; R. M. Wald, General Relativity (U. of Chicago, Chicago, 1984), Section 5.2.

[6] W. G. V. Rosser, An Introduction to Statistical Physics (Halsted Press, New York, 1985), Chapter 9; A. H. Carter, Classical and Statistical Thermodynamics (Prentice Hall, Upper Saddle River, NJ, 2001), Sections 18.1 and 18.2.

[7] M. S. Turner and M. White, "CDM models with a smooth component", Phys. Rev. D 56, R4439-R4443 (1997); V. Silveira and I. Waga, "Cosmological properties of a class of $\Lambda$ decaying cosmologies", Phys. Rev. D56, 4625-4632 (1997); R. R. Caldwell, R. Dave, and P. J. Steinhardt, "Cosmological imprint of an energy component with general equation of state", Phys. Rev. Lett. 80, 1582-1585 (1998); P. M. Garnavich, et. al., "Supernova limits on the cosmic equation of state", Astrophys. J. 509, $74-79$ (1998); J. P. Ostriker and P. J. Steinhardt, reference 2 .

[8] P. J. E. Peebles, "Tests of cosmological models constrained by inflation"”, Astrophys. J. 284, 439-444 (1984).

[9] See, for example, the plot of the age of the universe as a function of $\Omega_{m 0}$ and $\Omega_{v 0}$ in D. Goldsmith, reference 2, Figure 7.2, page 88.

[10] S. Weinberg, "The cosmological constant problem", Rev. Mod. Phys. 61, 1-23 (1989).

[11] E. W. Kolb and M. S. Turner, The Early Universe (Addison - Wesley, Redwood City, CA, 1990), Section 3.2.

[12] B. P. Schmidt, et. al., "The high-z supernova search: Measuring cosmic deceleration and global curvature of the universe using type 1a supernovae", Astrophys. J. 507, 46-63 (1988); S. Perlmutter, et. al. (The Supernova Cosmology Project), "Measurements of omega and lambda from 42 high-redshift supernovae", Astrophys. J. 517, 565-586 (1999); A. G. Riess, "The case for an accelerating universe from supernovae", Pub. Astron. Soc. Pac. 112, 1284-1299 (2000).

[13] G. C. McVittie, General relativity and cosmology (U. of Illinois, Urbana, 1965), Chapter 8; S. M. Carroll and W. H. Press, "The cosmological constant", Annu. Rev. Astron. Astrophys. 30, 499-542 (1992); J. A. Peacock, Cosmological physics (Cambridge U., Cambridge, 1999), Chapter 3.

[14] S. Weinberg, reference 1, Chapter 15, Section 3. 